\title{
Genetic Identification of Multiple Biological Roles Associated with the Capsid Protein of Satellite Panicum Mosaic Virus
}

\author{
Wenping Qiu and Karen-Beth G. Scholthof \\ Department of Plant Pathology and Microbiology, Texas A\&M University, College Station 77843, U.S.A. \\ Accepted 13 September 2000.
}

Satellite panicum mosaic virus (SPMV), an 824nucleotide, positive-sense, single-stranded RNA virus, depends on Panicum mosaic virus (PMV) for replication and spread in host plants. Compared with PMV infection alone, symptoms are intensified and develop faster on millet plants infected with SPMV and PMV. SPMV encodes a 157 amino acid capsid protein $(\mathrm{CP})(17.5 \mathrm{kDa})$ to encapsidate SPMV RNA and form $T=1$ satellite virions. The present study identifies additional biological activities of the SPMV CP, including the induction of severe chlorosis on proso millet plants (Panicum miliaceum cv. Sunup or Red Turghai). Initial deletion mutagenesis experiments mapped the chlorosis-inducing domain to amino acids 50 to 157 on the C-terminal portion of the SPMV CP. More defined analyses revealed that amino acids 124 to 135 comprised a critical domain associated with chlorosis induction and virion formation, whereas the extreme $C$ terminal residues 148 to 157 were not strictly essential for either role. The results also demonstrated that the absence of SPMV CP tended to stimulate the accumulation of defective RNAs. This suggests that the SPMV CP plays a significant role in maintaining the structural integrity of the full-length satellite virus RNA and harbors multiple functions associated with pathogenesis in SPMV-infected host plants.

Satellite panicum mosaic virus (SPMV) depends on its helper virus Panicum mosaic virus (PMV) (genus Panicovirus; family Tombusviridae) for replication and systemic spread in host plants (Scholthof 1999). PMV has a singlestranded, positive-sense RNA (ssRNA) genome of 4,326 nucleotides (nt) that harbors six genes (Turina et al. 1998). The 5 '-proximal p48 and p112 open reading frames (ORF) encode the core components of the viral replicase that are essential for replication of PMV and SPMV. Through the characterization of a set of PMV mutants, it was determined that the expression of the PMV capsid protein (CP) ORF (p26) and three other smaller ORFs (p8, p6.6, and p15) are necessary to support local and systemic movement of the viral complex in

Corresponding author: K.-B. G. Scholthof; Telephone: +1-979-845-8265; Fax: +1-979-845-6483; E-mail: kbgs@acs.tamu.edu

Current address of W. Qui: Department of Fruit Science, Southwest Missouri State University, Mountain Grove 65711, U.S.A. millet plants (Turina et al. 1998; Turina et al. 2000).

When coinoculated with its helper virus on millet host plants, SPMV intensified the severity of leaf symptoms, caused severe stunting and lack of seed set, and was associated with an approximately 1-day acceleration of systemic transport of the viral complex compared with inoculation with PMV alone (Scholthof 1999). The SPMV genome is composed of an 824-nt ssRNA (Masuta et al. 1987; Qiu and Scholthof 2000), encoding a 157 amino acid (aa) CP (17.5 $\mathrm{kDa})$ from an ORF that initiated from a start codon at nucleotide 88 and terminated at nucleotide 561 (Masuta et al. 1987). In vitro deletion mutagenesis of an infectious SPMV cDNA clone and characterization of SPMV-defective RNAs (DRNAs) that spontaneously accumulated in vivo revealed that the CP ORF is not essential for replication and movement of the satellite virus (Qiu and Scholthof 2000). Therefore, biological activities of the $17.5-\mathrm{kDa} \mathrm{CP}$, other than the encapsidation of the SPMV genomic RNA, remain largely unknown.

This study was initiated to investigate the potential role of SPMV CP in symptom elicitation and, subsequently, to map the functional domains to elucidate the mechanism of the synergistic interaction between SPMV and PMV. The results revealed that SPMV CP plays a crucial role in the induction of severe chlorosis on millet host plants, and this phenotype mapped to residues 50 to 157 on the C-proximal portion of the SPMV CP ORF. The dissection of this region by mutation analyses revealed overlapping domains on the $\mathrm{CP}$ that were associated with chlorotic symptoms and virion formation. The data also showed that in the absence of the $17.5-\mathrm{kDa} \mathrm{CP}$, SPMV was prone to accumulate D-RNAs in the infected plants.

\section{RESULTS}

SPMV CP modulates viral symptoms.

Visual symptoms of systemic virus infection on millet plants were monitored throughout a 3-week period following inoculation. Whereas subtle differences were difficult to evaluate on foxtail millet plants (Setaria italica cv. German Strain R), distinctive symptoms induced by the SPMV-type strain and its derived mutants (Fig. 1) on proso millet plants (Panicum miliaceum cv. Sunup or Red Turghai) were readily noticed. Symptoms on PMV-infected proso millet plants presented as a barely visible vein clearing and mild chlorotic mottle. In contrast, when the SPMV-type strain was coinocu- 
lated with PMV, a severe chlorotic mottle and bleaching effect on the leaves were frequently observed (Fig. 2).

To elucidate the possible contribution of the SPMV CP in its synergism with PMV (Scholthof 1999), two stop codons were introduced immediately downstream of the $\mathrm{CP}$ start codon on the SPMV-type strain cDNA to generate mutant SPMV-91 (Qiu and Scholthof 2000) (Fig. 1). In the mixed infection with PMV, SPMV-91 RNA replicated and moved through millet plants in the absence of the 17.5-KDa CP. Unexpectedly, the SPMV-91 mutant expressed a very low level of a small protein that was serologically related to the SPMV
CP. This protein is referred to as the $10-\mathrm{kDa} \mathrm{CP}$ (Qiu and Scholthof 2000). The expression of the 10-KDa protein likely occurs by translational reinitiation at an in-frame start codon at nucleotide 235 (gggAUGg), representing SPMV Cproximal amino acids 50 to 157 (Fig. 1).

In comparison with the SPMV-type strain, SPMV-91 induced a milder chlorotic mottle on the systemically infected leaves of proso millet plants (Fig. 2). This observation suggested that the chlorotic phenotype induced by SPMV infection was associated with $\mathrm{CP}$ expression. To further investigate the activity of SPMV CP as a symptom determinant, SPMV-

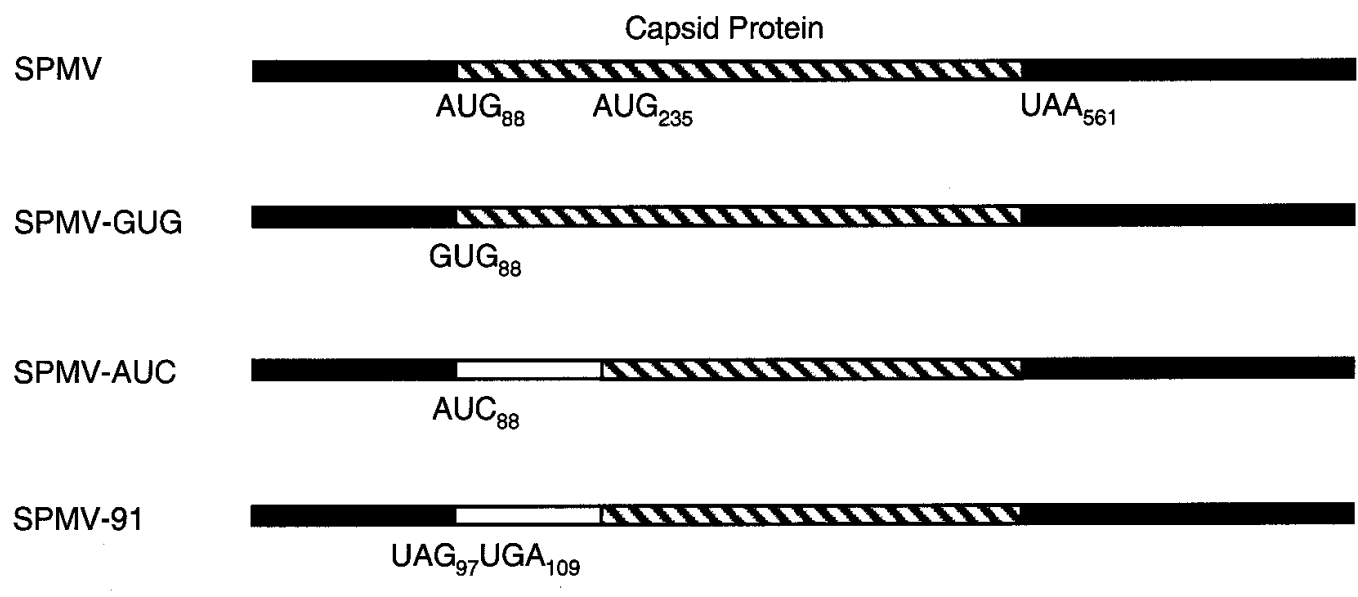

SP14

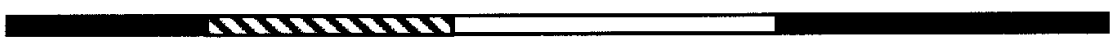

SP15

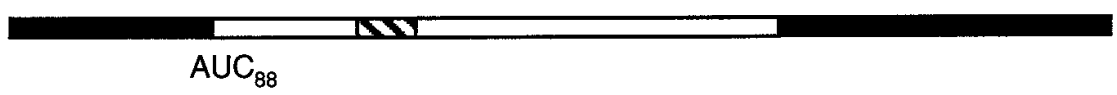

SP16

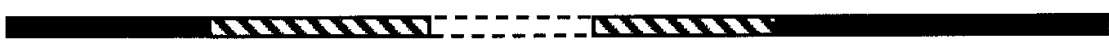

SP17

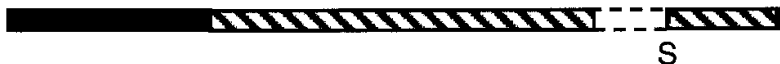

SP18

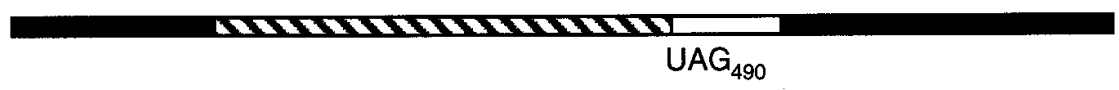

SP19

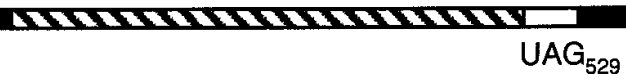

Fig. 1. Schematic representation of the RNA genome and the capsid protein (CP) coding regions of Satellite panicum mosaic virus (SPMV)-type strain and its derived mutants. Positions of the SPMV CP start codon (AUG) at nucleotide 88, a second in-frame start codon at nucleotide 235, and the original stop codon at nucleotide 561 are indicated on the SPMV cDNA map and occur on each of the other constructs, unless otherwise noted. A noncanonical start codon (GUG) and noninitiating codon (AUC) at nucleotide 88 were introduced on SPMV-GUG and SPMV-AUC mutants, respectively. Stop codons were introduced at nucleotides 97 and 109 on mutant SPMV-91. Mutants SP18 and SP19 are identified by amber stop codons (UAG) at nucleotides 490 and 529, respectively. The SPMV CP open reading frame (ORF) is depicted by a striped rectangle. Black rectangles denote the $5^{\prime}$ and $3^{\prime}$ untranslated regions. Nonexpressed regions of the $\mathrm{CP}$ in the respective mutants are indicated by white rectangles. White rectangles with dashed lines indicate portions inside the CP coding region that were deleted in SP16 and SP17 while retaining the in-frame downstream portions of the CP ORF. Amino acid 136 in the mutant SP17 changed from proline to serine (S). 
GUG was constructed to convert the authentic CP AUG start codon at nucleotide 88 to a noncanonical GUG start codon (Fig. 1). Immunoblots of total proteins from SPMV-GUGinfected millet plants probed with an antiserum specific to SPMV CP indicated that the switch from AUG to GUG decreased the accumulation of the $17.5-\mathrm{kDa} \mathrm{CP}$ by approximately $80 \%$, with a concomitant increase of the $10-\mathrm{kDa} \mathrm{CP}$ (Qiu and Scholthof 2000). This indicated that the downstream in-frame start codon at nucleotide 235 was recognized more efficiently on the SPMV-GUG RNA than on the SPMV-91 RNA, as predicted by the ribosome leaky scanning model (Kozak 1995; Qiu and Scholthof 2000). The combination of a decrease in the accumulation of the $17.5-\mathrm{kDa} \mathrm{CP}$ and an increase of the $10-\mathrm{kDa} \mathrm{CP}$ resulted in chlorotic blotches or vivid stripes with bleaching, on the systemically infected proso millet leaves. This feature distinguished SPMV-GUG infections as intermediate compared with the severe SPMV-type strain symptoms and the mild SPMV-91-infection phenotype (Fig. 2). These results indicated that the dosage of the 10- and 17.5-kDa CP affected the symptom intensity in the infected millet plants.

To determine the contribution of the $10-\mathrm{kDa} \mathrm{CP}$ to the induction of the bleaching symptoms, SPMV-AUC was created to change the authentic AUG start codon at nucleotide 88 to an AUC codon. The intent was to further reduce the expression of the 17.5-kDa CP and enhance the accumulation of the 10-kDa CP (Fig. 1 and Table 1). As predicted, an immunoblot assay of total plant proteins showed that SPMV-AUC-infected
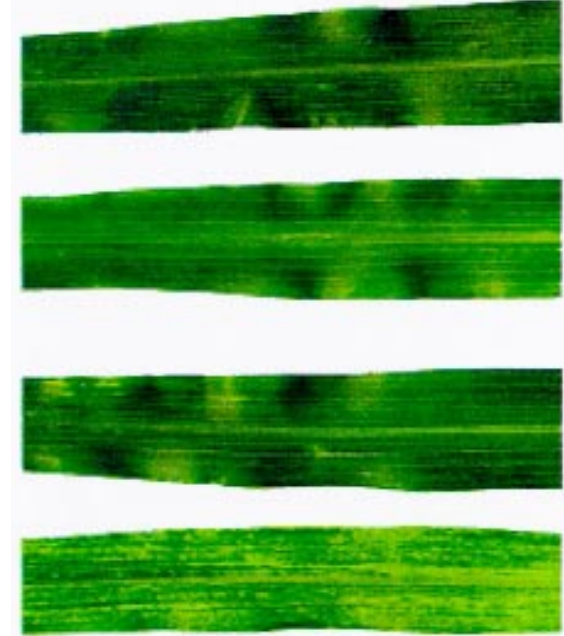

SP19

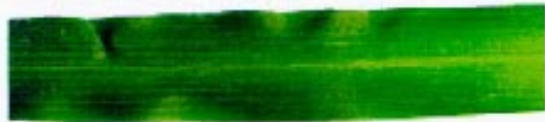

PMV

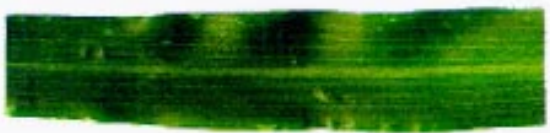

Mock

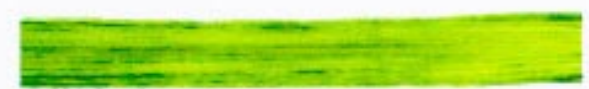

SPMV

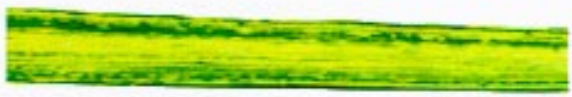

SPMV-GUG

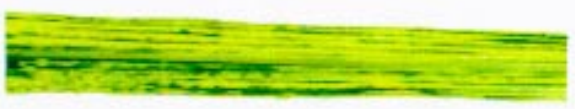

SPMV-AUC

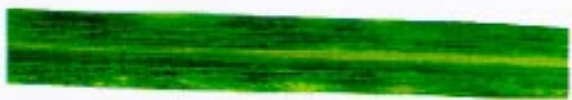

SPMV-91

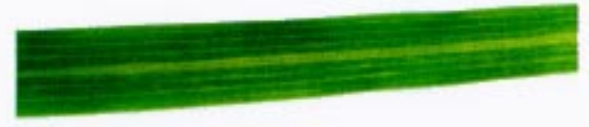

SP14

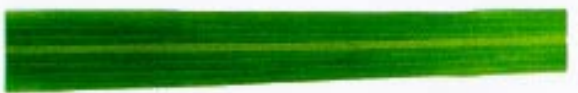

SP15

Fig. 2. Phenotypic comparisons of Satellite panicum mosaic virus (SPMV)-type strain, SPMV-GUG, SPMV-AUC, SPMV-91, and SPMV capsid protein deletion mutants (SP14-19) on the upper noninoculated leaves of proso millet plants (Panicum miliaceum cv. Sunup) coinoculated with Panicum mosaic virus (PMV). PMV-infected and mock-inoculated leaves are included for comparison. Leaves were photographed 3 weeks postinoculation.

Table 1. Genetic and biological characteristics of Satellite panicum mosaic virus (SPMV)-type strain and its derivative mutants

\begin{tabular}{|c|c|c|c|c|c|}
\hline Constructs & Amino acids deleted & $\mathbf{R N A}^{\mathbf{a}}$ & Capsid protein $(\mathbf{C P})^{\mathbf{b}}$ & Virion $^{\mathrm{c}}$ & Chlorosis $^{\mathrm{d}}$ \\
\hline SPMV & & gRNA & wt & + & + \\
\hline SPMV-AUC & 1 to 49 & gRNA & $\operatorname{tr}$ & - & + \\
\hline SP14 & 1 to 49,74 to 157 & g/D-RNA & - & - & - \\
\hline SP15 & 74 to 157 & gRNA & - & - & - \\
\hline SP16 & 76 to 124 & g/D-RNA & - & - & - \\
\hline SP17 & 124 to 135 & g/D-RNA & $\operatorname{tr}$ & - & - \\
\hline SP18 & 136 to 157 & D-RNA & - & - & - \\
\hline SP19 & 148 to 157 & g/D-RNA & $\operatorname{tr}$ & + & + \\
\hline
\end{tabular}

a gRNA indicates that only the genome-sized SPMV RNA was detected; D-RNA indicates that SPMV-defective RNAs predominated; g/D-RNA indicates the coexistence of gRNA and D-RNAs.

${ }^{\mathrm{b}}$ Summarized immunoblot data indicating that the 17.5-kDa SPMV CP (wt), a truncated form of SPMV CP (tr), or no detectable CP (-) accumulated in the inoculated plants.

${ }^{c}+$ and - indicates the presence or absence of SPMV virions on the whole virion gels assayed for SPMV CP and RNA.

${ }^{\mathrm{d}}+$ indicates a chlorotic symptom phenotype on the upper noninoculated leaves; - indicates no chlorosis. Symptoms were recorded 3 weeks postcoinoculation with Panicum mosaic virus and either SPMV RNA or its derivatives on proso millet plants (Panicum miliaceum cv. Sunup). 
millet plants accumulated high levels of the $10-\mathrm{kDa} \mathrm{CP}$, whereas the $17.5-\mathrm{kDa} \mathrm{CP}$ was not detected (Fig. 3). The symptoms on millet plants infected with the SPMV-AUC mutant were much the same as those induced by infections with the SPMV-GUG mutant (Fig. 2), supporting the conclusion that the C-terminal portion of the $\mathrm{CP}$ represents a major symptom determinant. Another line of evidence that the 10$\mathrm{kDa} C \mathrm{P}$ was associated with chlorosis induction was provided by frameshift mutants SP14 and SP15. These constructs were designed to abolish the expression of the 10-kDa SPMV CP (Fig. 1). Nucleotides 308 to 311 were deleted from the SPMVtype strain cDNA to create SP14, which was predicted to express an 8 -kDa protein containing $\mathrm{N}$-terminal residues 1 to 73 of SPMV CP. Mutant SP15, which was derived from mutant SPMV-AUC, had nucleotides 308 to 309 deleted to express a putative 26-aa polypeptide from the AUG at nucleotide 235 (Fig. 1 and Table 1). The truncated protein encoded by SP14 was detected by an in vitro translation assay in wheat germ extract with a predicted molecular mass of $8 \mathrm{kDa}$, but the truncated protein by SP15 migrated slightly higher than that predicted for a $2.9-\mathrm{kDa}$ protein (Fig. 4). SP14 and SP15 mutants did not produce detectable proteins in systemically infected millet plants (Fig. 3A), although both mutants were replicated and spread by PMV (Fig. 3B). The symptom phenotypes induced by coinfection of PMV with SP14 and SP15 mutants were similar to those observed during infection of

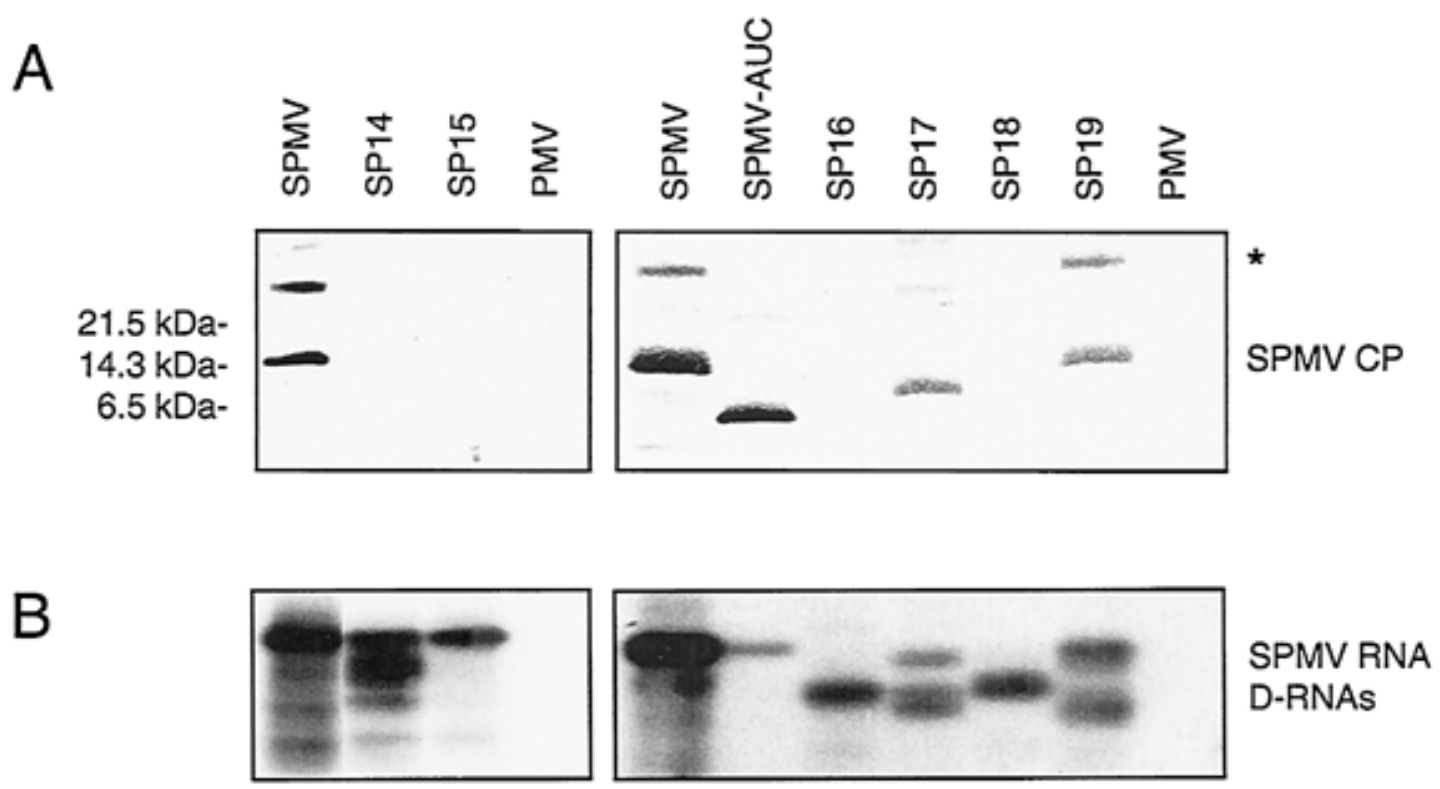

Fig. 3. Accumulation of the Satellite panicum mosaic virus (SPMV) capsid protein (CP) and RNA in infected proso millet plants (Panicum miliaceum cv. Sunup) 14 days postinoculation with Panicum mosaic virus alone or with SPMV or its derivatives. A, Immunoblot probed with the polyclonal antibody specific for SPMV CP. The asterisk indicates the relative location of a putative dimer of each CP form. Positions of protein molecular weight markers are indicated on the left side of the panel. B, RNA blot assay with a ${ }^{32} \mathrm{P}$-labeled SPMV cDNA-specific probe that also detects the associated D-RNAs. Lanes representing SPMV-type strain and derived mutants are indicated above the blots.

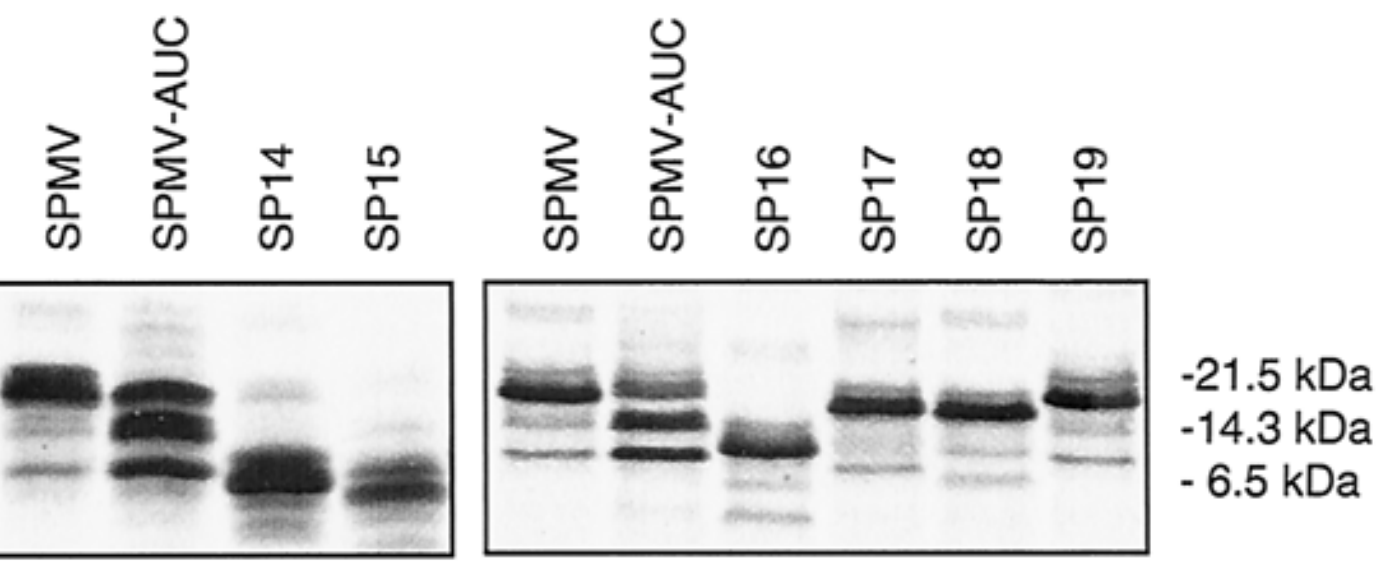

Fig. 4. In vitro translation assay in wheat germ extract of the Satellite panicum mosaic virus (SPMV)-type strain and its derived mutants showing the expression of wild-type (17.5-kDa) and truncated forms of the SPMV capsid protein (CP). The location of the protein molecular weight markers are indicated in $\mathrm{kDa}$ on the right side of the panel. Although in vitro translation assays with SPMV-AUC transcripts produced three proteins of 10-, 17.5-, and approximately $13 \mathrm{kDa}$, only the 10-kDa form of the SPMV CP was detected in vivo. Designation of the SPMV-type strain and each derived mutant is listed above the blot. 
plants with constructs that did not express the 17.5- or $10-\mathrm{kDa}$ CP (Fig. 2). Therefore, the results collectively support the conclusion that the $10-\mathrm{kDa}$ protein encompassing the $\mathrm{C}$ proximal SPMV CP residues 50 to 157 is necessary and sufficient for the induction of chlorosis upon coinfection with PMV in proso millet.

\section{Characteristics of SPMV C-terminal deletion mutants.}

The above biological assays implied that the $10-\mathrm{kDa}$ portion of the SPMV CP was the critical domain for the induction of chlorotic symptoms on proso millet plants coinfected with PMV. To dissect the putative chlorosis-inducing domains, four C-terminal CP mutants were generated from the SPMV-type strain cDNA (Fig. 1). Mutant SP16 had codons for residues 76 to 124 deleted to generate an $11-\mathrm{kDa}$ form of the SPMV CP, and mutant SP17 was predicted to express a $16-\mathrm{kDa} \mathrm{CP}$ following the deletion of a region encoding residues 124 to 135 . Both deletion mutants retained the ability to produce the extreme C-proximal portion of the $\mathrm{CP}$ (Fig. 1 and Table 1). The introduction of a stop codon at nucleotide 490 (SP18) or 529 (SP19) resulted in the expression of a $15-$ or $16-\mathrm{kDa}$ truncated SPMV CP, respectively (Fig. 1 and Table 1). In vitro translation assays with uncapped transcripts in wheat germ extract showed that the mutants produced C-terminal truncated proteins of the predicted molecular mass (Fig. 4). In addition, each of the truncated proteins was recognized by antiserum against SPMV CP, although the CP expressed in vitro from the SP16 and SP18 transcripts was weakly recognized (data not shown). Immunoblot assays of total proteins from infected proso millet plants detected the $17.5-\mathrm{kDa} \mathrm{CP}$ produced by the SPMV-type strain, the $10-\mathrm{kDa} \mathrm{CP}$ translated from SPMVAUC, and the truncated CPs from SP17 and SP19 (Fig. 3A). CP derivatives that were predicted to be translated from the SP16 or SP18 mutants, however, were not detected in planta (Fig. 3A).

Total RNAs were extracted from the upper, noninoculated leaves of proso millet plants coinfected with PMV and either the SPMV-type strain, SPMV-AUC, or four SPMV CP Cterminal deletion mutants (SP16-19) at 14 days postinoculation (dpi) and then subjected to an RNA blot assay. The
SPMV mutants producing the C-terminal-truncated $\mathrm{CP}$ derivatives were competent for replication and systemic movement (Fig. 3B). In addition, the SP16, SP17, and SP18 mutants induced a very mild symptom phenotype, similar to an infection by PMV alone. In comparison, the SP19 mutant elicited a chlorotic mottle, although the symptoms were milder than those elicited by the SPMV-type strain (Fig. 2). Taken together, the data indicate that the C-terminal portion of the $\mathrm{CP}$ is an important determinant for the chlorotic phenotype in millet plants coinfected with PMV and SPMV.

\section{Putative domains on the SPMV CP involved in virion formation.}

Millet leaves infected with the SPMV-type strain, SPMVAUC, and the SPMV CP mutants (SP14-19) were assayed for SPMV and PMV virions. The virions were resolved on duplicate $1 \%$ agarose gels to allow transfer to a nylon membrane for a hybridization assay to detect encapsidated RNA (Fig. 5) and to a nitrocellulose membrane for immunodetection of SPMV CP (not shown). As expected, PMV virions were detected in all infected millet plants (Fig. 5A), but SPMV virions were associated only with wild-type and SP19 infections. RNAs from the type strain and SP19 were detected on the whole virion gel at a position that corresponded to the migration of SPMV virions (Fig. 5B). Mutants SP15 and SP17 were competent for replication and long-distance spread (Fig. 3), but they did not form SPMV virions and were not associated with PMV virions (Fig. 5). RNAs of the mutant SPMV-AUC, SP14, SP16, or SP18 did not comigrate to a position of SPMV virions (Fig. 5B), as predicted by their inability to produce $\mathrm{CP}$. RNAs from SP14, SP16, and SP18, however, colocalized predominantly with PMV virions (Fig. 5B), suggesting that this set of mutant SPMV RNAs were tightly bound to or possibly encapsidated by PMV CP.

\section{Correlation between the absence of the 17.5-kDa CP and the accumulation of SPMV D-RNAs.}

RNA hybridization assays revealed that D-RNAs (Qiu and Scholthof 2000) accumulated in millet plants coinfected with

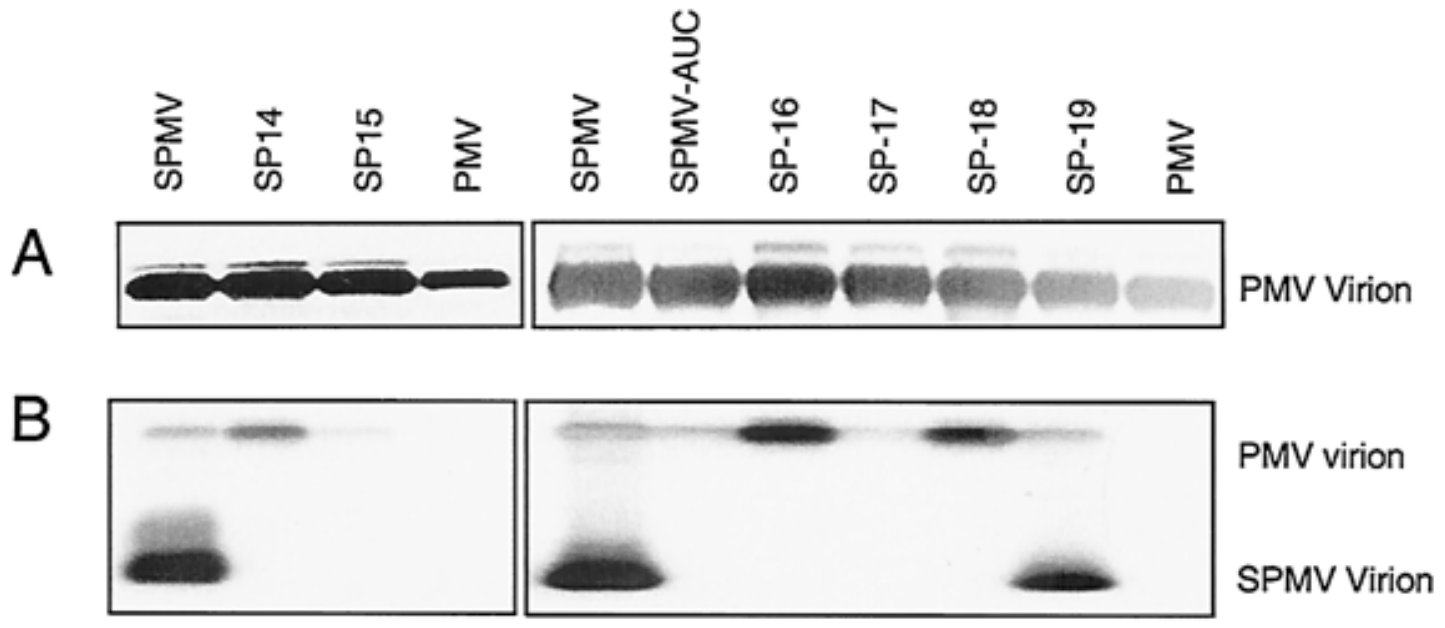

Fig. 5. Encapsidation assay of Satellite panicum mosaic virus (SPMV) capsid protein and its truncated forms. A, Photographs of ethidium bromidestained gels are shown as a negative image to demonstrate the presence of Panicum mosaic virus (PMV) virions in all samples. B, Virions were transferred to nylon membrane for detection of SPMV RNA with a ${ }^{32} \mathrm{P}-$ labeled SPMV cDNA-specific probe. SPMV-specific RNAs from mutants SP14, SP16, and SP18 migrated to the same position as PMV virions. Labels for SPMV-type strain and its derived mutants are indicated above the panels. 
PMV and the SPMV CP deletion mutants SP14, SP17, SP18, and SP19 (Table 1 and Fig. 3B). Progeny RNAs derived from SP16 (deletion of nucleotides 308 to 457 on the SPMV cDNA) migrated at the predicted position, but it was not clear whether additional small deletions had occurred. To further characterize these derivatives, representative D-RNAs were isolated from plants infected with the mutants SP16, SP18, and SP19 and used as templates to generate the corresponding cDNAs. Two representative cDNA clones derived from each D-RNA sample were sequenced. The D-RNA cDNA sequences are presented schematically to allow for comparisons of the retained RNA elements (Fig. 6).
In addition to the original deletion of nucleotides 308 to 457 on the SP16 mutant, the regions covering nucleotides 136 to 463 and nucleotides 145 to 463 were deleted in the SP16 DRNA-9 and D-RNA-12, respectively. Neither of the SP16derived D-RNAs was predicted to possess a functional ORF because stop codons did not occur on the cDNAs. Three regions were removed at different locations from the mutant SP18 in the D-RNA-13 and D-RNA-17 generations (Fig. 6). The SP18 D-RNA-13 potentially encodes a 9-aa polypeptide, whereas SP18 D-RNA-17 codes for a putative 65-aa protein. Three regions were deleted on SP19 D-RNA-20, and two regions were excised from SP19 D-RNA-21. Interestingly, six

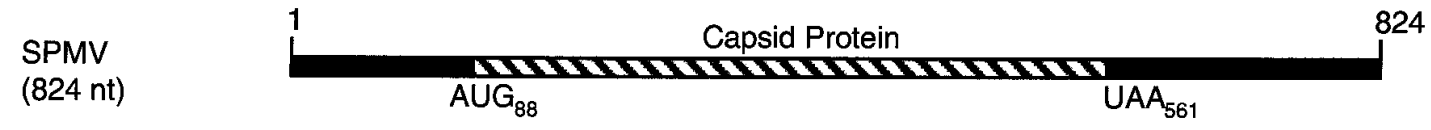

SP16

$307 \quad 458$

(674 nt)

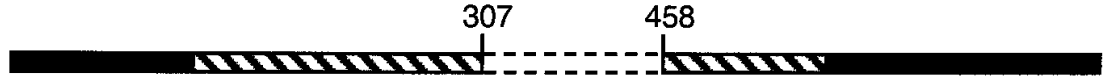

SP16 D-RNA-9

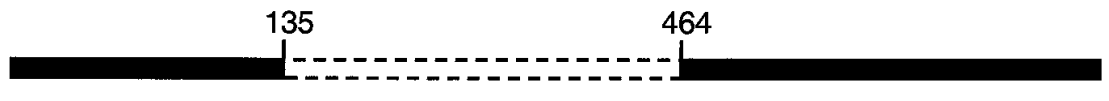

(496 nt)

SP16 D-RNA-12

(505 nt)

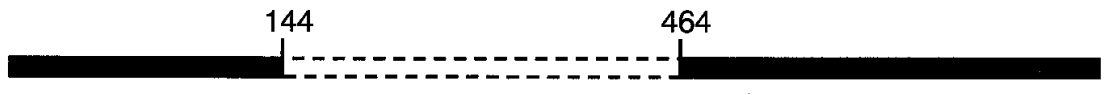

SP18 D-RNA-13

(531 nt)

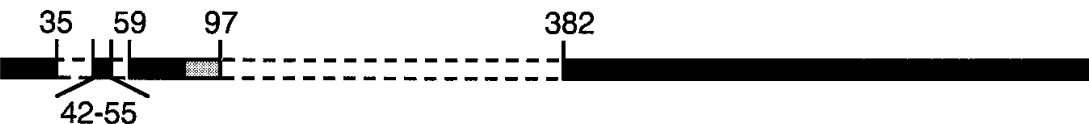

SP18 D-RNA-17

(511 nt)

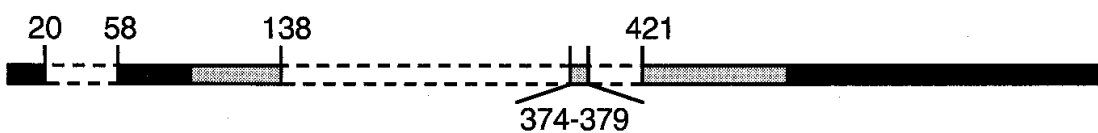

SP19 D-RNA-20

(415 nt)

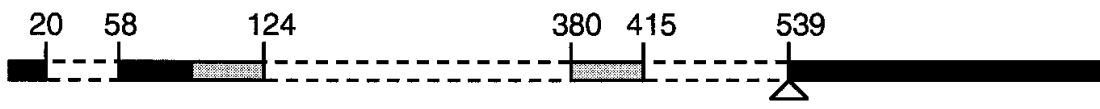

SP19 D-RNA-21

(405 nt)

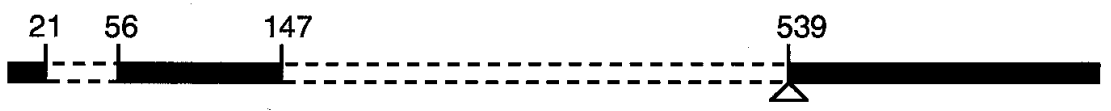

Fig. 6. Schematic diagram of representative Satellite panicum mosaic virus (SPMV) D-RNA cDNAs isolated from proso millet plants (Panicum miliaceum cv. Sunup) infected with Panicum mosaic virus (PMV) and SP16, SP18, or SP19. The designation of each D-RNA cDNA and its size in nucleotides is indicated. SP16 is included for comparison with two derived deletion mutants, D-RNA-9 and D-RNA-12. Striped rectangles represent the CP coding region in the SPMV-type strain and the mutant SP16. Gray rectangles denote the putative coding regions in the D-RNAs. Filled rectangles represent RNA fragments retained in the D-RNAs with the nucleotide positions relative to the SPMV-type strain RNA genome. Rectangles with dashed lines designate deleted portions on the cDNA. RNA transcripts from the D-RNA cDNA clones indicated in bold letters were selected for infectivity assays and were shown to be infectious on millet plants when coinoculated with PMV RNA. The triangle indicates the presence of six extra nucleotides (CCTAGG) in SP19 D-RNA cDNAs. 
extra nucleotides (CCTAGG) were inserted at position 539 on the two D-RNAs derived from SP19, as determined from the cDNA sequence. SP19 D-RNA-20 was predicted to encode a 27-aa polypeptide, whereas SP19 D-RNA-21 did not encode a functional ORF (no stop codon). Although deletions occurred at different locations, all SPMV-derived D-RNAs retained the first $20 \mathrm{nt}$ on the $5^{\prime}$ untranslated region (UTR) and nucleotides 539 to 824 on the $3^{\prime}$ UTR.

\section{Infectivity assays of the SPMV D-RNAs.}

RNA transcripts were made from one of each pair of the SP16, SP18, and SP19 D-RNA cDNA constructs and mixed with PMV transcripts to inoculate proso millet plants. RNA blot analyses of total RNA from upper noninoculated leaves demonstrated that SP16 D-RNA-9, SP18 D-RNA-13, and SP19 D-RNA-20 were replicated by PMV and moved systemically, proving that they are biologically active in millet plants (Fig. 7). The millet plants coinfected by PMV and SPMV D-RNAs showed mild symptoms, similar to those on plants infected with PMV alone.

\section{DISCUSSION}

Exacerbation of symptom severity on plants caused by satellite viruses during coinfection with a helper virus is an intriguing biological feature of this group of "molecular parasites" (Dodds 1999; Scholthof et al. 1999). For example, the mild symptoms on millet plants infected with PMV are greatly enhanced by coinfection with SPMV (Scholthof 1999). Similarly, symptoms induced by Tobacco mosaic virus (TMV-U2) on jalapeño and pimiento peppers were enhanced by coinfection with satellite tobacco mosaic virus (STMV), resulting in abundant bright-yellow patches on the upper noninoculated leaves (Rodriguez-Alvarado et al. 1994). The cellular aberrations associated with STMV infections included modification of the mitochondria, membranous vesicles, and a lack of TMV-characteristic X-bodies (Kim et al. 1989). The severe chlorosis induced by coinfections of SPMV and PMV also is associated with obvious cytopathic effects on host organelles (Wilson 1974). It has not been determined, however, which viral factors (the RNA and/or the expressed protein) or processes (replication, translation, and/or movement) of satellite viruses contribute to the synergistic interactions with their respective helper viruses.

The satellite virus $\mathrm{CP}$ is a candidate for inducing proteinmediated effects in the infected cell. Genetic analyses of SPMV, STMV, and Satellite tobacco necrosis virus showed that the $\mathrm{CP}$ genes of these satellite viruses can be deleted without abolishing replication and systemic movement (Bringloe et al. 1998; Qiu and Scholthof 2000; Routh et al. 1995; Routh et al. 1997). The results from the present study show that although SPMV CP is dispensable, its expression is associated with the development of severe chlorosis on proso millet plants and the SPMV RNA has minimal effects on symptom expression (Figs. 1 and 2; Table 1). Therefore, the SPMV CP can be grouped with plant virus CPs that harbor multifaceted biological activities, including encapsidation, activation of replication, elicitation of a hypersensitive response, involvement in systemic movement, and determination of the symptom phenotype (Canto et al. 1997; Kong et al. 1997; Taraporewala and Culver 1996; Tenllado and Bol 2000;
Vaewhongs and Lommel 1995; Wang and Simon 2000).

The first SPMV CP-associated activity discussed in this study involves its effect on symptomatology. The forced expression of the $10-\mathrm{kDa}$ C-terminal portion of the SPMV CP from the SPMV-GUG and SPMV-AUC mutants induced chlorosis and bleaching on millet leaves that was more striking than the symptoms induced by the type strain. In contrast, mutants SP14 and SP15, which fail to produce the 10-kDa CP, induced a mild chlorosis (Fig. 2). The intensity of chlorosis was positively correlated with the dosage of the $10-\mathrm{kDa} \mathrm{CP}$, as illustrated by the comparison of symptoms induced by the mutants SPMV-AUC and SPMV-91 (Qiu and Scholthof 2000) (Fig. 2). These results support the conclusion that the Cproximal portion of the SPMV CP, covering residues 50 to 157, represents the "chlorosis-inducing" domain. The deletion of residues 124 to 135 (mutant SP17) abolished virion formation and induced only a mild chlorosis (Figs. 2, 4, and 5), indicating that this region may contain a critical motif for one or both of these features. The roles of the SPMV amino acid residues 76 to 124 and 136 to 148 in virion formation and symptom development are unclear. The truncated SPMV CPs, which were predicted to be produced by mutants SP16 and SP18, were not detected by immunoblot assays, probably as a result of the accumulation of D-RNAs or instabilities of SPMV CP subunits with deletions in the central portion (Fig. 3). However, extreme C-terminal truncations from amino acids 148 to 157 on mutant SP19 did not significantly affect chlorosis induction or virion formation (Figs. 2, 4, and 5).

Another result of this study included a tentative identification of an N-terminal domain for encapsidation. The 10-kDa $\mathrm{CP}$ produced by SPMV-AUC did not form virions (Fig. 5), suggesting that the N-terminal amino acids 1 to 49 harbor a critical domain for the SPMV CP and RNA interaction. This observation is in agreement with predictions that an $\mathrm{N}$ terminal arginine-rich motif (ARM) of the SPMV CP interacts with SPMV genomic RNA and resides in the interior of the virion (Ban et al. 1995; Ban and McPherson 1995). Our data

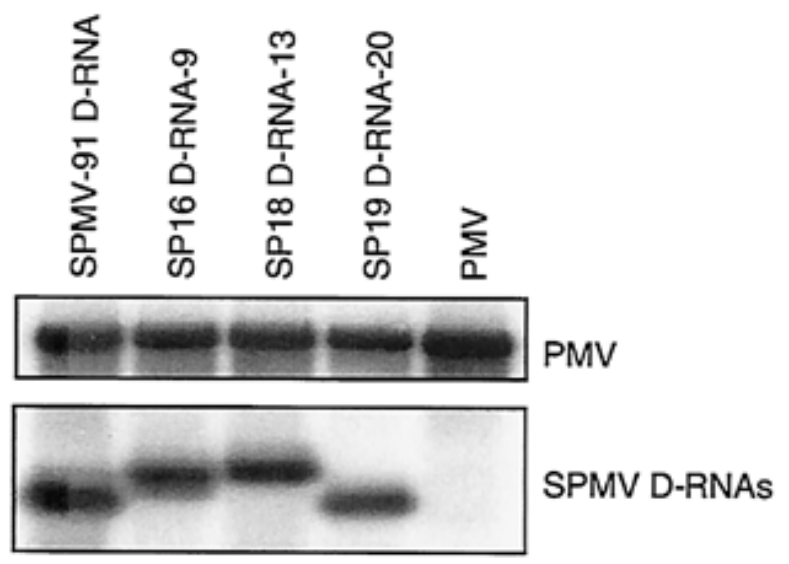

Fig. 7. RNA blot assay of proso millet plants (Panicum miliaceum cv. Sunup) infected with Panicum mosaic virus (PMV) transcript alone or in combination with each of four SPMV-derived D-RNA transcripts, SPMV-91 D-RNA, SP16 D-RNA-9, SP18 D-RNA-13, and SP19 DRNA-20. The RNA blot was first hybridized with a ${ }^{32} \mathrm{P}-$ labeled SPMV cDNA-specific probe to detect the presence of D-RNAs (bottom panel) and subsequently with a PMV-specific probe to show the presence of PMV in all samples (top panel). SPMV-91 D-RNA (Qiu and Scholthof 2000) was included as positive control. 
is supported by studies with a $20-\mathrm{kDa}$ protein encoded by Bamboo mosaic virus satellite RNA (satBaMV RNA). The SPMV CP and the satBaMV 20-kDa protein share $46 \%$ identity and conservation of an ARM from amino acids 1 to 12 (SPMV: MAPKRSRRSNER; satBaMV: MVRRRNRRQRSR; conserved alignment in bold) (Masuta et al. 1987; Tsai et al. 1999). In vitro assays revealed that the RNA-protein interactions between satBaMV RNA and its 20-kDa protein are dependent on the intact ARM (Lin et al. 1996; Tsai et al. 1999). Functional analyses of the Brome mosaic virus and Cucumber mosaic virus CPs also demonstrate that an $\mathrm{N}$ proximal 19-aa ARM has a critical role in CP-viral RNA interactions and virion formation (Rao and Grantham 1996; Schmitz and Rao 1998). Collectively, these data suggest that the SPMV CP ARM is an RNA binding domain that is required for encapsidation of SPMV RNA by SPMV CP.

Another intriguing biological process that was affected by SPMV CP involved the generation and accumulation of DRNAs. SPMV D-RNAs accumulated de novo in infected millet plants following coinoculation of PMV with SPMV mutants SP14, SP16, SP17, SP18, and SP19, and none of these mutants expressed the $17.5-\mathrm{kDa}$ CP (Fig. 3B). The DRNAs represent discrete colinear regions derived from the full-length SPMV RNA (Fig. 6). This result indicates that expression of the $17.5-\mathrm{kDa}$ SPMV CP can inhibit the accumulation of D-RNAs. Thus, it is unclear if the SPMV CP actively participates as a "fidelity censor" in the process of its SPMV RNA synthesis to ensure synthesis of full-length, wildtype SPMV genome or if it influences a cellular RNA surveillance pathway. For example, SPMV CP may act as a putative suppressor of defense-related posttranscriptional gene silencing (Voinnet et al. 1999) to prevent the accumulation of aberrant RNAs that could be the D-RNA progenitors in millet plants infected with PMV and SPMV. Alternatively, the observed preferential accumulation of D-RNAs and the reduced accumulation of wild-type SPMV RNA in the absence of SPMV CP expression could be the result of more favored packaging, or perhaps spread, of D-RNAs by PMV CP. This hypothesis is supported by our observation that the SP14, SP16, and SP18 mutant RNAs, which exclusively accumulate D-RNAs, migrate to the same position as PMV virions (Fig. 5). This indicates that the D-RNAs either bind PMV CP or are packaged into helper virions.

One unusual observation was the lack of D-RNAs in mixed PMV infections with mutants SPMV-AUC and SP15, which do not produce wild-type CP (Fig. 3). These constructs differ by only a few nucleotides from SPMV-GUG and SP14 mutants that also are devoid of CP synthesis but do stimulate DRNA accumulation. This implies that there are either very refined structural features determining whether an RNA is predisposed toward D-RNA formation and/or a critical interaction with a host protein, perhaps a component of the translational machinery, that could influence this process. There is precedent for the role of cellular factors that are associated with the translational machinery in maintaining the replication of D-RNAs (Spagnolo and Hogue 2000; White et al. 1992).

Our results support the conclusion that the SPMV CP is a crucial factor in inducing severe disease in mixed infections of PMV and SPMV on millet plants, whereas the SPMV RNA has minimal influence on the symptom phenotype. Amino acids 1 to 49 play an important role in SPMV CP-RNA inter- actions and virion formation (SPMV-AUC). Residues 50 to 157 are sufficient for the induction of the severe chlorosis symptoms. Amino acids 124 to 135 (SP17) are involved in virion formation and chlorosis, but residues 148 to 157 (SP19) are not essential for those functions. In the absence of the SPMV CP, the SPMV D-RNAs have a tendency to emerge in infected millet plants, which suggests a significant role for SPMV CP in maintaining the integrity of its homologous RNA genome, although other unidentified effects also may influence this process.

\section{MATERIALS AND METHODS}

\section{Host plants and inoculation.}

Foxtail millet and proso millet were grown in the greenhouse $\left(28\right.$ to $\left.30^{\circ} \mathrm{C}\right)$ or in a growth chamber $\left(28^{\circ} \mathrm{C}, 14 \mathrm{~h}\right.$ of light; $24^{\circ} \mathrm{C}, 10 \mathrm{~h}$ of dark). Four millet plants at the three-leaf stage were mechanically inoculated by mixing equal volumes of uncapped RNA transcripts (approximately $6 \mu \mathrm{g}$ ) and RNA inoculation buffer $\left(0.05 \mathrm{M} \mathrm{K}_{2} \mathrm{HPO}_{4} ; 0.05 \mathrm{M}\right.$ glycine; $1 \%$ bentonite; $1 \%$ celite, $\mathrm{pH}$ 9.0), as described previously (Turina et al. 1998). Inoculated plants were maintained overnight at room temperature before being transferred to the greenhouse or growth chamber. The symptoms on inoculated millet plants were documented and photographed 2 or 3 weeks postinoculation. Each bioassay was repeated three times with independently synthesized PMV and SPMV RNA transcripts.

\section{Site-directed oligonucleotide mutagenesis and deletion mutagenesis of the SPMV cDNA.}

Single-stranded DNA was generated from the SPMV cDNA phagemid with standard protocols (Kunkel et al. 1987). The construction of mutants SPMV-GUG and SPMV-91 was described previously (Qiu and Scholthof 2000). The primer SPMV-AUCBamHI (5'-CGAGGGATCCTCCTGATCGCTCCTAAGC-3'; the BamHI site is underlined and the ATC modifying the authentic AUG CP start codon is in bold) was designed to create mutant SPMV-AUC. The primer SPMV 490stopStuI (5'-CCAAGGGTGCCTAGGCCTCCAGCAATACC-3'; the amber stop codon is in bold and the StuI site is underlined) was used to introduce a stop codon at nucleotide 490 to create mutant SP18, and a StuI site at nucleotide 491 was used for convenient screening of mutants and subsequent construction of a deletion mutant SP17. The primer SPMV529stopNheI (5'-GGGTTGCTTTTAGCTAGCGCCTAGCGAG-3'; the amber stop codon is in bold and the NheI site is underlined) was used to introduce a stop codon at nucleotide 529 to generate the mutant SP19 with a NheI site at nucleotide 531 to facilitate screening by restriction enzyme analysis.

Deletion mutants SP14 and SP15 were made by BssHII digestion of the SPMV-type strain and SPMV-AUC cDNAs, respectively, and treatment with a DNA polymerase I large fragment (Klenow enzyme). Mutant SP16 was constructed by the deletion of nucleotides 308 to 457 through the digestion of the SPMV-type strain cDNA with BssHII (at nucleotide 307) and $\mathrm{MscI}$ (at nucleotide 455) followed by treatment with Klenow enzyme. Deletion mutant SP17 was generated by deleting nucleotides 458 to 493 through the digestion of mutant SP18 with $M s c$ I and StuI. The predicted modifications and deletions of SPMV-type strain and SPMV-AUC cDNAs were confirmed 
by sequencing the relevant plasmids. The deletion mutants SP16 and SP17 retained the authentic CP ORF with predicted truncations.

\section{Construction of SPMV D-RNA cDNA clones.}

The SPMV D-RNAs were isolated from infected plants by a gel-purification procedure following total RNA extraction, as previously described (Qiu and Scholthof 2000). The purified RNAs were used as templates in the reverse transcription polymerase chain reaction (RT-PCR) with the use of the following primers: a reverse SPMV primer $\left(5^{\prime}\right.$-CTGCAGATCTGGGTCCTAGGAGGGGGAGACGC-3'; PstI site is underlined and $B g l I I$ is in bold) complementary to the $3^{\prime}$-terminal nucleotides 803 to 824 was used for first-strand cDNA synthesis; a forward SPMV primer (5'-GGATCCTAATACGACTCACTATAGGGTATTCCACGCTAGCAAC-3'; BamHI site is underlined) contained the first $20 \mathrm{nt}$ in the $5^{\prime}$ end of SPMV, which was linked with an upstream T7 RNA polymerase promoter sequence (in bold letters). Following RT-PCR of DRNAs, the blunt-end cDNA products were ligated into the SmaI site of pUC119 and sequenced.

\section{In vitro transcription and translation.}

A CsCl-purified plasmid containing the PMV cDNA (Turina et al. 1998) was linearized with EcoICR1 for a fulllength template. Plasmids containing the SPMV-type strain cDNA (Monis et al. 1992), mutagenized SPMV cDNAs, and the SPMV D-RNA cDNA clones were linearized with $B g l I I$. Uncapped RNA transcripts were generated by T7 RNA polymerase (Gibco BRL Co., Gaithersburg, MD, U.S.A.) with approximately $0.5 \mu \mathrm{g}$ of linearized DNA template, as previously described (Qiu and Scholthof 2000).

Approximately $200 \mathrm{ng}$ of RNA transcript was used as a template in $10 \mu \mathrm{l}$ of in vitro wheat germ translation reactions consisting of $1 \mu \mathrm{l}$ of ${ }^{35} \mathrm{~S}$-methionine and other reagents, as recommended by the supplier (Promega, Madison, WI, U.S.A.), with $110 \mathrm{mM}$ potassium acetate to optimize translation. The reaction mixtures were incubated at $25^{\circ} \mathrm{C}$ for 2.5 to $3 \mathrm{~h}$. The resultant proteins from half of the reaction mix were denatured and resolved on $12.5 \%$ sodium dodecyl sulfatepolyacrylamide gels by electrophoresis (Turina et al. 1998). The proteins were transferred from the gel to nitrocellulose membrane for immunodetection by SPMV CP-specific antiserum. Following immunodetection, the same blot was exposed to X-ray film to detect the ${ }^{35} \mathrm{~S}$-methionine-labeled protein products.

\section{Minipurification of virions and whole-virion assays.}

Symptomatic leaves were harvested from virus-infected millet plants at $14 \mathrm{dpi}$, and $0.5 \mathrm{~g}$ of leaf tissue was ground in 2

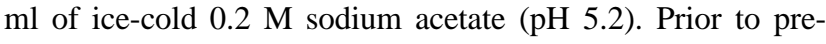
cipitation of the virions in $12 \%$ polyethylene glycol (PEG)8000 , the leaf extracts were transferred to $1.5-\mathrm{ml}$ tubes and incubated at $37^{\circ} \mathrm{C}$ for $30 \mathrm{~min}$ to utilize the endogenous plant RNases to degrade any unprotected RNAs, including host cell RNA and unbound viral RNAs (Jupin et al. 1990). The leaf extracts were then centrifuged at $10,000 \times g, 4^{\circ} \mathrm{C}$, for $10 \mathrm{~min}$. The supernatant was transferred to an empty tube, and the virions were precipitated on ice for $30 \mathrm{~min}$ by the addition of $12 \%$ PEG-8000 and $0.3 \mathrm{M} \mathrm{NaCl}$. The virions were then collected by centrifugation at $10,000 \times g, 4^{\circ} \mathrm{C}$, for $10 \mathrm{~min}$. The pellet was resuspended in $0.05 \mathrm{M}$ sodium acetate $(\mathrm{pH} 5.5)$, and a second precipitation was performed overnight at $4{ }^{\circ} \mathrm{C}$ with the same concentration of PEG and $\mathrm{NaCl}$, as described above. The virions were collected by centrifugation at 10,000 $\times g, 4^{\circ} \mathrm{C}$, for $10 \mathrm{~min}$ and subsequently resuspended in $50 \mu \mathrm{l}$ of $0.05 \mathrm{M}$ sodium acetate, $\mathrm{pH} 5.5$. A $1 \%$ Tris-glycine agarose gel in $2 \times$ Tris-glycine buffer $(10 \mathrm{mM}$ Tris, $76 \mathrm{mM}$ glycine, $\mathrm{pH}$ 8.0) was used to resolve $5 \mu \mathrm{l}$ of resuspended virions. Whole virions were transferred to nylon or nitrocellulose membranes (Micron Separations Inc., Westborough, MA, U.S.A.) for the PMV- or SPMV-specific RNA and CP assays.

\section{RNA and protein analyses.}

Total RNAs or proteins were extracted from four bulked, systemically infected millet leaves at $14 \mathrm{dpi}$ and assayed for the presence of viral RNAs or CPs, as previously described (Qiu and Scholthof 2000; Scholthof et al. 1993). PMV, SPMV-type strain, and mutant SPMV RNAs were detected by hybridizing the RNA blots with ${ }^{32} \mathrm{P}-\mathrm{dCTP}$-labeled PMV- or SPMV-specific DNA probes (Turina et al. 1998). The protein blots were incubated with either rabbit polyclonal anti-SPMV $\mathrm{CP}$ antiserum or rabbit polyclonal anti-PMV $\mathrm{CP}$ antiserum to detect the presence of viral CP proteins, as described in previous studies (Scholthof 1999; Turina et al. 1998).

\section{ACKNOWLEDGMENTS}

We thank Herman Scholthof, Bénédicte Desvoyes, and Jeff Batten for their helpful discussions and critical reviews of this manuscript. The Texas Agricultural Experiment Station (H-8388) and USDA-NRI Competitive Grant 99-35303-3714 supported this research.

\section{LITERATURE CITED}

Ban, N., and McPherson, A. 1995. The structure of satellite panicum mosaic virus at 1.9^̊ resolution. Nat. Struct. Biol. 2:882-890.

Ban, N., Larson, S. B., and McPherson, A. 1995. Structural comparison of the plant satellite viruses. Virology 214:571-583.

Bringloe, D. H., Gultyaev, A. P., Pelpel, M., Pleij, C. W. A., and Coutts, R. H. A. 1998. The nucleotide sequence of satellite tobacco necrosis virus strain $\mathrm{C}$ and helper-assisted replication of wild-type and mutant clones of the virus. J. Gen. Virol. 79:1539-1546.

Canto, T., Prior, D. A. M., Hellwald, K.-H., Oparka, K. J., and Palukaitis, P. 1997. Characterization of cucumber mosaic virus. IV. Movement protein and coat protein are both essential for cell-to-cell movement of cucumber mosaic virus. Virology 237:237-248.

Dodds, J. A. 1999. Satellite tobacco mosaic virus. Curr. Top. Microbiol. Immunol. 239:145-157.

Jupin, I., Richards, K., Jonard, G., Guilley, H., and Pleij, C. W. A. 1990. Mapping of sequences required for productive replication of beet necrotic yellow vein virus RNA 3. Virology 178:273-280.

Kim, K.-S., Valverde, R. A., and Dodds, J. A. 1989. Cytopathology of satellite tobacco mosaic virus and its helper virus in tobacco. J. Ultrastruct. Mol. Struct. Res. 102:196-204.

Kong, Q., Wang, J., and Simon, A. E. 1997. Satellite RNA-mediated resistance to turnip crinkle virus in Arabidopsis involves a reduction in virus movement. Plant Cell 9:2051-2063.

Kozak, M. 1995. Adherence to the first-AUG rule when a second AUG codon follows closely upon the first. Proc. Natl. Acad. Sci. USA 92:2662-2666.

Kunkel, T. A., Roberts, J. D., and Zakour, R. A. 1987. Rapid and efficient site-specific mutagenesis without phenotypic selection. Methods Enzymol. 154:367-382.

Lin, N.-S., Lee, Y.-S., Lin, B.-Y., Lee, C.-W., and Hsu, Y.-H. 1996. The open reading frame of bamboo mosaic potexvirus satellite RNA is not essential for its replication and can be replaced with a bacterial gene. Proc. Natl. Acad. Sci. USA 93:3138-3142. 
Masuta, C., Zuidema, D., Hunter, B. G., Heaton, L. A., Sopher, D. S., and Jackson, A. O. 1987. Analysis of the genome of satellite panicum mosaic virus. Virology 159:329-338.

Monis, J., Sopher, D. S., and Jackson, A. O. 1992. Biologically active cDNA clones of panicum mosaic virus satellites. (Abstr.) Phytopathology 82:1175.

Qiu, W. P., and Scholthof, K.-B. G. 2000. In vitro- and in vivo-generated defective RNAs of satellite panicum mosaic virus define cis-acting RNA elements required for replication and movement. J. Virol. 74:2247-2254.

Rao, A. L. N., and Grantham, G. L. 1996. Molecular studies on bromovirus capsid protein. II. Functional analysis of the amino-terminal arginine-rich motif, and its role in encapsidation, movement, and pathology. Virology 226:294-305.

Rodríguez-Alvarado, G., Kurath, G., and Dodds, J. A. 1994. Symptom modification by satellite tobacco mosaic virus in pepper types and cultivars infected with helper tobamoviruses. Phytopathology 84:617621.

Routh, G., Dodds, J. A., Fitzmaurice, L., and Mirkov, T. E. 1995. Characterization of deletion and frameshift mutants of satellite tobacco mosaic virus. Virology 212:121-127.

Routh, G., Ngon A Yassi, M., Rao, A. L. N., Mirkov, T. E., and Dodds, J. A. 1997. Replication of wild-type and mutant clones of satellite tobacco mosaic virus in Nicotiana benthamiana protoplasts. J. Gen. Virol. 78:1271-1275.

Schmitz, I., and Rao, A. L. N. 1998. Deletions in the conserved aminoterminal basic arm of cucumber mosaic virus coat protein disrup virion assembly but do not abolish infectivity and cell-to-cell movement. Virology 248:323-331.

Scholthof, H. B., Morris, T. J., and Jackson, A. O. 1993. The capsid protein gene of tomato bushy stunt virus is dispensable for systemic movement and can be replaced for localized expression of foreign genes. Mol. Plant-Microbe Interact. 6:309-322.

Scholthof, K.-B. G. 1999. A synergism induced by satellite panicum mosaic virus. Mol. Plant-Microbe Interact. 12:163-166.

Scholthof, K.-B. G., Jones, R. W., and Jackson, A. O. 1999. Biology and structure of plant satellite viruses activated by icosahedral helper viruses. Curr. Top. Microbiol. Immunol. 239:123-143.

Spagnolo, J. F., and Hogue, B. G. 2000. Host protein interactions with the $3^{\prime}$ end of bovine coronavirus RNA and the requirement of the poly(A) tail for coronavirus defective genome replication. J. Virol. 74:5053-5065.

Taraporewala, Z. F., and Culver, J. N. 1996. Identification of an elicitor active site within the three-dimensional structure of the tobacco mosaic tobamovirus coat protein. Plant Cell 8:169-178.

Tenllado, F., and Bol, J. F. 2000. Genetic dissection of the multiple functions of alfalfa mosaic virus coat protein in viral RNA replication, encapsidation, and movement. Virology 268:29-40.

Tsai, M.-S., Hsu, Y.-H., and Lin, N.-S. 1999. Bamboo mosaic potexvirus satellite RNA (satBaMV RNA)-encoded P20 protein preferentially binds to satBaMV RNA. J. Virol. 73:3032-3039.

Turina, M., Maruoka, M., Monis, J., Jackson, A. O., and Scholthof, K.B. G. 1998. Nucleotide sequence and infectivity of a full-length cDNA clone of panicum mosaic virus. Virology 241:141-155.

Turina, M., Desvoyes, B., and Scholthof, K.-B. G. 2000. A gene cluster encoded by panicum mosaic virus is associated with virus movement. Virology 266:120-128.

Vaewhongs, A. A., and Lommel, S. A. 1995. Virion formation is required for the long-distance movement of red clover necrotic mosaic virus in movement protein transgenic plants. Virology 212:607-613.

Voinnet, O., Pinto, Y. M., and Baulcombe, D. C. 1999. Suppression of gene silencing: A general strategy used by diverse DNA and RNA viruses of plants. Proc. Natl. Acad. Sci. USA 96:14147-14152.

Wang, J., and Simon, A. E. 2000. 3'-end stem-loops of the subviral RNAs associated with turnip crinkle virus are involved in symptom modulation and coat protein binding. J. Virol. 74:6528-6537.

White, K. A., Bancroft, J. B., and Mackie, G. A. 1992. Coding capacity determines in vivo accumulation of a defective RNA of clover yellow mosaic virus. J. Virol. 66:3069-3076.

Wilson, W. J. 1974. Ultrastructural analysis of millet plants infected with St. Augustine decline. Ph.D. diss. Texas A\&M University, College Station, U.S.A 